\title{
Soothing Efficacy and Tolerability of a Skin Care Product Containing Live Lactobacillus rhamnosus Bacteria and Berry Seed Oils on Atopic Dermatitis Lesions
}

\author{
Beata Imko-Walczuk ${ }^{1}$, Aleksandra Taraszkiewicz ${ }^{1}$, Annika Mäyrä2 \\ ${ }^{1}$ Dermscan Poland, Gdansk, Poland \\ ${ }^{2}$ Gutguide Ltd., Helsinki, Finland \\ Email: ata@dermscan.pl, annika.mayra@gutguide.fi
}

How to cite this paper: Imko-Walczuk, B., Taraszkiewicz, A. and Mäyrä, A. (2019) Soothing Efficacy and Tolerability of a Skin Care Product Containing Live Lactobacillus rhamnosus Bacteria and Berry Seed Oils on Atopic Dermatitis Lesions. Journal of Cosmetics, Dermatological Sciences and Applications, 9, 83-93.

https://doi.org/10.4236/jcdsa.2019.92007

Received: January 23, 2019

Accepted: April 6, 2019

Published: April 9, 2019

Copyright $\odot 2019$ by author(s) and Scientific Research Publishing Inc. This work is licensed under the Creative Commons Attribution International License (CC BY 4.0).

http://creativecommons.org/licenses/by/4.0/

\begin{abstract}
Background: Atopic dermatitis $(\mathrm{AD})$ is a chronic pruritic inflammatory skin disease characterized by a reduced microbiome diversity which favours the skin colonization with pathogenic bacteria, mainly with Staphylococcus aureus. A novel approach to the management of several skin diseases, including AD, involves the use of specific beneficial probiotic bacteria aimed at restoring the skin microbiome balance and decreasing the skin colonization with pathogens. Objective: This study assessed the soothing efficacy and tolerability of a new probiotic-based skin care product combining two live Lactobacillus rhamnosus strains and berry seed oils on eczematous lesions in subjects with atopic skin. Patients and Methods: Twenty-five adults with atopic skin and presenting at least one inflammatory lesion applied the product on the defined lesion for 28 days, once to twice daily. Severity of eczema signs (EASI score) and intensity of pruritus (VAS) were assessed, and digital images were captured following treatment. Cutaneous tolerability and subject satisfaction were also investigated. Results: A statistically significant decrease in mean EASI score was observed after 28 days $(-27 \%, \mathrm{p}=0.011)$. A visible improvement in skin aspect was observed in $67 \%$ of the subjects on week 4 . On average, the intensity of pruritus decreased by $57 \%$ as compared to baseline ( $\mathrm{p}=$ 0.011 ). This effect was observed in $86 \%$ of the subjects. No relevant adverse reactions were observed following treatment with the skin care product and the majority of the subjects appreciated the product for its general characteristics, properties, and efficacy. Conclusion: The results suggest that this skin care product containing two live Lactobacillus rhamnosus strains and berry seed oils could be used for the treatment of eczematous lesions in subjects with atopic skin.
\end{abstract}




\section{Keywords}

Atopic Dermatitis, Lactobacillus rhamnosus, Probiotic, Berry Seed Oils, Pruritus

\section{Introduction}

Atopic dermatitis (AD) or atopic eczema is a chronic pruritic inflammatory skin disease affecting $1 \%-10 \%$ of adults worldwide and typically characterized by intensely pruritic, xerotic lesions in the skin with erythema, oedema and/or papules, exudate, excoriation, and lichenification [1] [2] [3] [4].

$\mathrm{AD}$ is usually associated with a personal or family history of type I allergies, allergic rhinitis and asthma [2]. The genetic predisposition can be exacerbated by several factors, including food allergy, skin infections, irritating clothes or chemicals, change in climate, and emotions [5].

The two major and interacting pathophysiological peculiarities of $\mathrm{AD}$ are a defective epidermal barrier and a cutaneous inflammation due to inappropriate immune responses to antigens encountered in the skin. The abnormalities of the epidermal structure and function are thought to be responsible for a decline in antimicrobial peptides production in the skin and a reduced microbiome diversity which favours the skin colonization with pathogenic bacteria [4] [6] [7]. Bacterial colonization with Staphylococcus aureus ( $S$. aureus) is the most common skin infection in $\mathrm{AD}$, affecting more than $90 \%$ of patients and occurring on both lesional and, to a lesser extent, non-lesional $\mathrm{AD}$ skin [8] [9]. S. aureus-derived proteases and enterotoxins further contribute to barrier disruption and inflammation, thus perpetuating exacerbations and chronification [10].

Management of $\mathrm{AD}$ symptoms is based on a multistep approach combining continuous epidermal barrier repair with emollients, avoidance of individual trigger factors, antimicrobial therapy for acute infections, and anti-inflammatory therapy with topical corticosteroids or calcineurin inhibitors [11]. As $S$. aureus plays a role in important aspects of $\mathrm{AD}$ pathogenesis, strategies to restore the microbiome diversity and decrease the skin colonization with $S$. aureus in patients with $\mathrm{AD}$ would likely help to reduce the frequency and severity of $\mathrm{AD}$ symptoms. Recently, the use of specific probiotic bacteria to restore the skin microbiome homeostasis has emerged as a novel approach to manage several skin diseases, including rosacea, acne, and $\mathrm{AD}$ [12]. Probiotics are Generally Regarded As Safe (GRAS determination) and therefore could potentially be used if they have therapeutic value.

When administered to AD patients, specific probiotic strains were shown to repair the lipidic skin barrier, restore the cutaneous microflora, and stimulate the skin immune system balance by favouring a Th1 cell response [12] [13] [14]. Only few studies have explored topical application of probiotics on the altered skin of AD patients [14] [15].

In the present study, we aimed to assess the soothing efficacy and tolerability 
of a new skin care product combining two live Lactobacillus rhamnosus strains and berry seed oils on eczematous lesions of subjects with atopic skin.

\section{Methods}

\subsection{Population}

This study was conducted on 25 healthy subjects aged at minimum 18 years, with a Fitzpatrick skin type I-IV and atopic skin with, at least, one inflammatory lesion on the body (including hands) and face.

Exclusion criteria included pregnant or nursing women or women planning to get pregnant during the study, subjects with a cutaneous pathology on the studied zone (except $\mathrm{AD}$ ), subjects having undergone a surgery under general anaesthesia within the previous month, individuals who have been excessively exposed to sunlight or UV-rays within the previous month, subjects who used anti-inflammatory products on the studied zones within the previous month, as well as subjects who used topical or systemic treatments during the previous weeks liable to interfere with the efficacy assessment of the studied product. All subjects enrolled in another clinical trial during the study period were also excluded from the study.

The subjects were allowed to use their usual cleansing products (e.g., soap, shower gel) and skin-care products (cream, lotion) during the whole study period, but not on the defined inflammatory lesion. Shaving or depilatory products were also allowed during the course of the study, except on visit days.

\subsection{Study Design}

This was a 4-week open-label clinical trial performed between February and June 2018 in "Dermscan Poland", a clinical research organization located in Gdansk (Poland).

The subjects were instructed to apply the product on the defined inflammatory lesion from baseline for a period of 4 weeks, once to twice daily (in the morning and/or in the evening) on clean and dry skin until complete penetration, as recommended by the manufacturer. Evaluation of the treatment was performed at baseline and at the end of week 4 (28 days).

The tested product (SkinGuide ${ }^{\mathrm{ru}}$ ) was supplied in a vial (Biodue Ltd., Italy) containing berry seed oils (1\% sea buckthorn oil and 1.5\% blackcurrant oil, provided by Aromtech Ltd., Finland) and a mixture of two freeze-dried probiotic strains: Lactobacillus rhamnosus LB21 CS and Lactobacillus rhamnosus SP1 CS (Sacco Ltd., Italy) in a final concentration of $1 \times 10^{9} \mathrm{CFU} / \mathrm{g}$. The strains were conserved in the vial cap to ensure bacteria stability for at least 18 months. The mechanism has been developed so that when turning the cap clockwise, the bacteria are released and mixed with oil. Four vials were supplied to the subjects (1 vial to be used per week); the viability of the strains after 7 days was confirmed in a previous study (Gutguide Ltd., internal data).

The study was conducted on a cosmetic product whose safety has been as- 
sured by the Sponsor. The European Directive 2001/20/CE, transposed in Polish Law (version of 07.08.2009, amended by the European Regulation of the European Parliament \#596/2009 dated on June 18, 2009-Dziennik Urzędowy, \#L188, page 14, dated on 18.7.2009) was not applicable. Therefore, this study did not require the Ethics Committee Approval and the Competent Authority Authorization.

\subsection{Efficacy Assessment}

The efficacy of the studied product was evaluated at baseline and after 4 weeks based on the following parameters.

\subsubsection{EASI Score}

The severity of eczema signs (erythema, infiltration and/or papulation, excoriation, and lichenification) on the defined lesion was assessed by the dermatologist with the Eczema Area Severity (EASI) index on a 4-point scale ( $0=$ none, $1=$ mild, $2=$ moderate, $3=$ severe). The EASI score was calculated as the sum of each of the 4 evaluated signs.

\subsubsection{VAS Score}

The subjects were asked to evaluate the severity of pruritus under dermatological control using a Visual Analogue Scale (VAS) from 0 (no pruritus) to 10 (severe pruritus).

\subsubsection{Lesion Imaging}

Digital images of the defined inflammatory lesion of all the subjects were captured with a Nikon ${ }^{\circledR}$ D90 digital camera. The macrophotographs were taken in standardized, indirect light. Aperture, speed and distance of the camera were also standardized.

\subsection{Assessment of Cutaneous Tolerability}

The possible adverse reactions were collected on an ongoing basis by analysing the daily log completed by the subjects. The subjects were also questioned about potential intolerance sensations felt or observed during the study.

\subsection{Self-Assessment Questionnaire}

Each subject of the study completed a questionnaire at the end of the study period on week 4 to evaluate the properties of the studied product and its global efficacy.

\subsection{Statistical Methods}

Descriptive statistics were calculated for each quantitative data: means, medians, minima, maxima, standard errors of the means (SEM), confidence intervals at 95\% (CI 95\%) of these values as well as variations $(\Delta)$ and percentage variations $(\Delta \%)$. A Shapiro-Wilk test $(\alpha=0.01)$ was used to determine normality of the differences. According to the result of the normality test, a Paired Student t-test 
or a Wilcoxon signed rank test was used, respectively, for normal and skewed distribution.

All differences were considered to be statistically significant at $\mathrm{p} \leq 0.05$. All analyses were performed using Microsoft Excel 2016 and PASW ${ }^{\circledR}$ statistics 25 $\left(\right.$ SPSS $\left.^{\circledR}\right)$.

\section{Results}

\subsection{Demographics and Baseline Characteristics}

Of the 25 subjects enrolled in the trial, 23 completed the whole study period (one subject dropped out from the study and another subject was lost to follow-up). Two subjects were excluded from the analysis due to major protocol violations decreasing the total number of participants to 21 subjects for efficacy and safety assessment. Most of the subjects were females (76\%) with an average age of 41 $( \pm 3)$ years. The majority of the subjects had a skin phototype of II $(78 \%)$ and presented a dry face (48\%) and body (52\%) skin type (Table 1$)$.

Table 1. Subject demographics and baseline characteristics.

\begin{tabular}{|c|c|}
\hline Variable & Value \\
\hline \multicolumn{2}{|l|}{ Age, years } \\
\hline $\operatorname{Mean}(S D)$ & $41( \pm 3)$ \\
\hline Range & {$[22 ; 69]$} \\
\hline \multicolumn{2}{|l|}{ Sex, n (\%) } \\
\hline Female & $17(76 \%)$ \\
\hline Male & $6(24 \%)$ \\
\hline \multicolumn{2}{|l|}{ Phototype, n (\%) } \\
\hline$I$ & 0 \\
\hline$I I$ & $18(78 \%)$ \\
\hline$I I I$ & $4(18 \%)$ \\
\hline$I V$ & $1(4 \%)$ \\
\hline \multicolumn{2}{|c|}{ Face skin type, n (\%) } \\
\hline Normal & $4(17 \%)$ \\
\hline Combination & $7(31 \%)$ \\
\hline Greasy & $1(4 \%)$ \\
\hline Dry & $11(48 \%)$ \\
\hline Very dry & 0 \\
\hline \multicolumn{2}{|c|}{ Body skin type, n (\%) } \\
\hline Normal & 0 \\
\hline Dry & $12(52 \%)$ \\
\hline Very dry & $11(48 \%)$ \\
\hline
\end{tabular}

$\mathrm{N}=23$. 


\subsection{Efficacy Results}

\subsubsection{EASI Score}

A statistically significant decrease in mean EASI score was observed following application of the skin care product on the defined inflammatory lesions for 28 days $(-27 \%, \mathrm{p}=0.011)$. A visible improvement in skin aspect was observed in $67 \%$ of the subjects on week 4 (Table 2 ).

\subsubsection{VAS Score}

After 28 days of treatment, the intensity of pruritus significantly decreased by $57 \%$ in average, as compared to baseline $(\mathrm{p}=0.011)$. This effect was observed in $86 \%$ of the subjects (Table 2 ).

\subsubsection{Lesion Imaging}

A significant improvement in the skin lesion was observed at the end of week 4 in several subjects and on various parts of the body. Figure 1 shows representative images of the best results obtained with three subjects.

\subsection{Cutaneous Tolerability}

Only 5 subjects experienced adverse events during the study. The most common

Table 2. EASI and VAS scores at baseline and after 4 weeks of treatment.

\begin{tabular}{lcccccc}
\hline & Baseline & $\begin{array}{c}\text { Week 4 } \\
\text { (day 28) }\end{array}$ & $\begin{array}{c}\text { Difference, } \\
\text { mean } \pm \text { SEM }\end{array}$ & $\begin{array}{c}\text { Difference, } \\
\text { percentage }\end{array}$ & $\begin{array}{c}\text { Percentage of subjects } \\
\text { with expected effect }\end{array}$ & p value \\
\hline EASI score & 4.3 & 3.1 & $-1.2 \pm 0.5$ & $-27 \%$ & $67 \%$ & 0.011 \\
VAS score & 5.5 & 2.4 & $-3.2 \pm 0.7$ & $-57 \%$ & $86 \%$ & $<0.001$ \\
\hline
\end{tabular}

$\mathrm{N}=23$; EASI: Eczema Area Severity Index; VAS: Visual Analogue Scale; SEM: Standard Error Mean. All changes were statistically significant $\mathrm{p} \leq 0.05$.

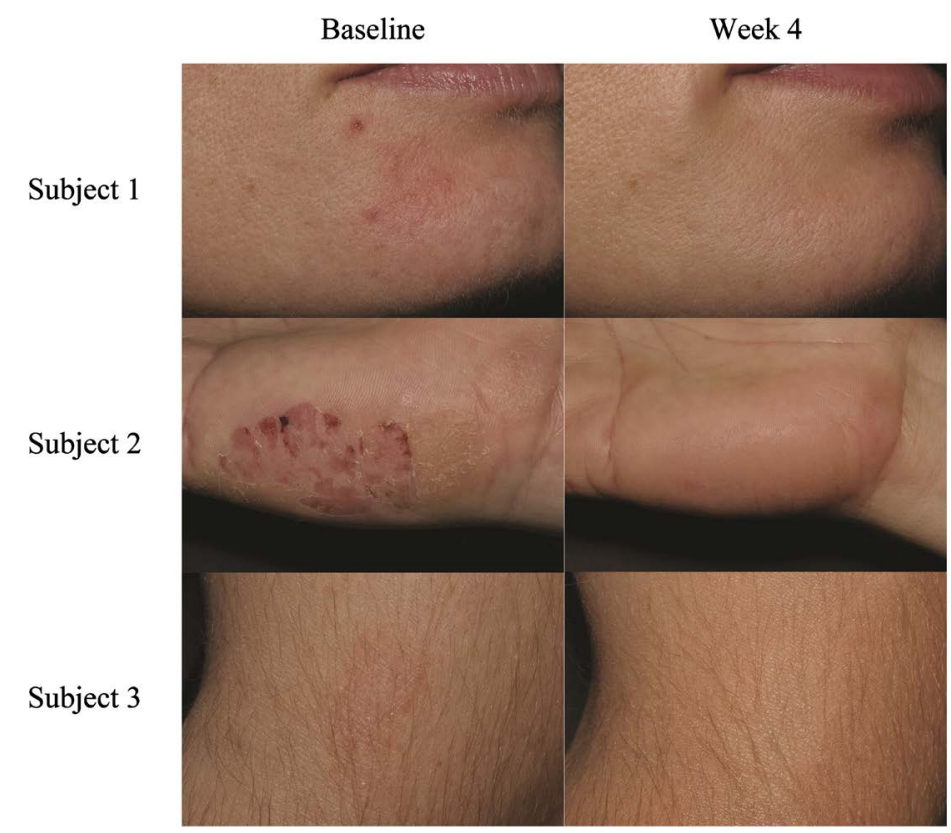

Figure 1. Clinical photographs of three subjects at baseline and after 4 weeks of treatment. 
manifestations likely to be related to the study product were itching, redness and burning sensations, which were mild in intensity and resolved shortly after topical application.

\subsection{Self-Assessment Questionnaire}

Overall, the majority of the subjects appreciated the product for its general characteristics and properties, but also for its efficacy (Figure 2).

The subjects reported that the product protects from external aggressions (69\%), leaves the skin moisturized (68\%) and reduces pruritus (64\%). Overall, $59 \%$ of the subjects declared that the product reduces discomfort sensations, leaves the skin comfortable and soothed.

\section{Discussion}

The altered skin barrier and immune dysfunction involved in $\mathrm{AD}$ pathogenesis are related with a lower microbial diversity on the eczematous areas, as well as an increase in $S$. aureus proportion during flare-ups [9]. Regimens that include antimicrobial therapies are associated with resistant bacteria emergence and removal of beneficial resident bacteria species due to their broad action on the entire skin microbiota [4] [7]. We hypothesized that re-establishing the skin microbiome balance by introducing beneficial bacteria strains might improve the aspect of xerotic lesions and the associated itching.

We evaluated the soothing efficacy of a probiotic-based skin care product in subjects presenting inflammatory lesions related to $\mathrm{AD}$. The present study demonstrated a clear soothing and anti-pruritic effect of the studied product, as assessed with the EASI and VAS scales. The product was well tolerated throughout the study period and its beneficial effects were recognized by the subjects.

Several studies have demonstrated the ability of specific commensal bacteria to improve symptoms of $\mathrm{AD}$, although most of the studies have used oral probiotics acting on the gut [14] [16] [17]. Application of topical probiotics to modulate the cutaneous microbiota has also been investigated with reportedly beneficial

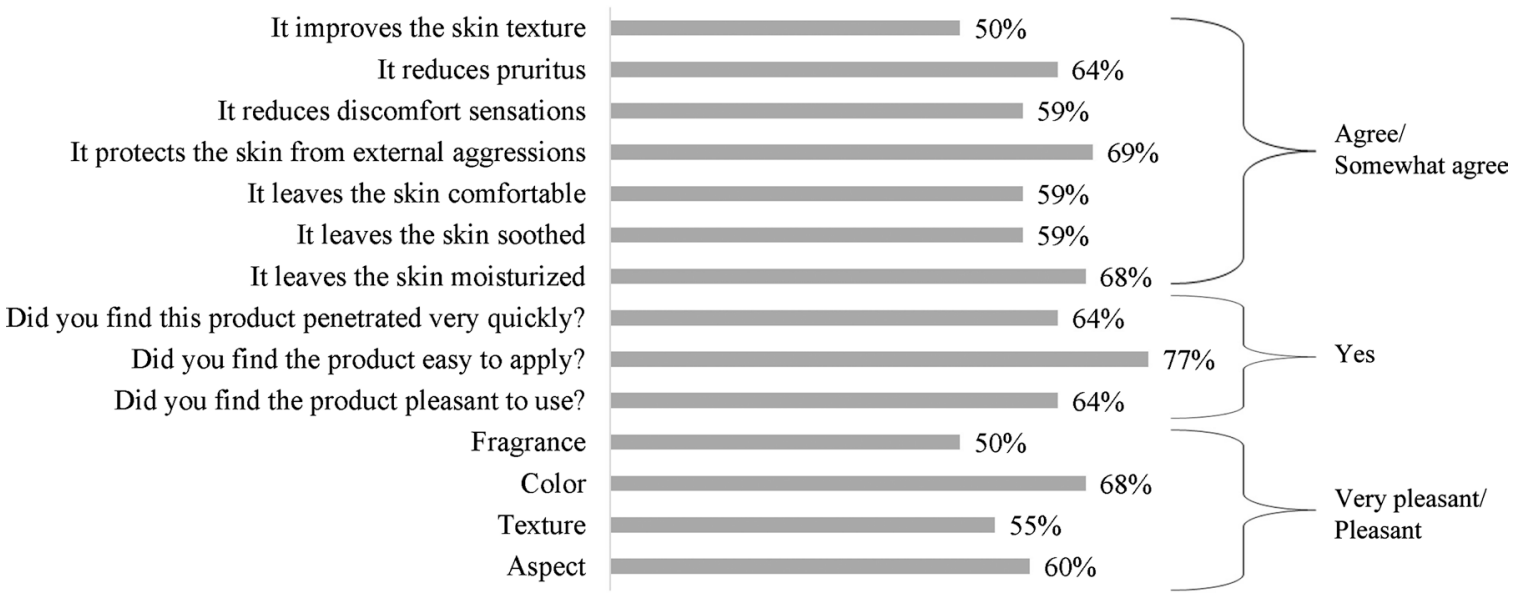

Figure 2. Self-assessment questionnaire responses at week 4. 
effects on the site of application. A lotion containing $5 \%$ of non-pathogenic Vitreoscilla filiformis lysate was assessed in patients with atopic eczema [18]. The lotion was found to significantly alleviate the signs and symptoms of atopic skin in the study population when applied twice daily on all $\mathrm{AD}$ skin lesions compared to the symmetrical $\mathrm{AD}$ lesions which received the placebo. The authors assumed that the beneficial effect might be due to a reduction in $S$. aureus counts and possibly a direct immunomodulatory effect on skin-associated immune responses. In another study, Gueniche and colleagues have reported the effect of a cream containing Bifidobacterium longum extract on reactive skin [19]. The authors found that subjects who applied the cream had a significant decrease in skin sensitivity and increase in skin resistance against physical and chemical aggression. The efficacy of a daily moisturizing lotion containing $0.3 \%$ Lactobacillus johnsonii NCC 533 inactivated by heat treatment (HT La1 lotion) was also investigated in patients with $\mathrm{AD}$ [20]. After 3 weeks of twice daily application to target AD lesions, HT La1 lotion significantly lowered $S$. aureus loads compared with the contralateral lesions that were untreated or treated with the patient's usual moisturizer. The control of $S$. aureus colonization was associated with a clinical statistically significant improvement in the treated AD lesions, as assessed by the SCORAD index.

Because probiotic effects are strain specific, careful selection of the strains is indispensable to ensure treatment efficacy. In the present study, we used a mixture of two Lactobacillus strains, namely Lactobacillus rhamnosus SP1 and Lactobacillus rhamnosus $\mathrm{LB}$. These two probiotic strains were chosen due to their interesting complementary features (adhesion capacities, metabolic substances, survival rates). We assumed that these two strains could potentially exert synergistic actions to improve $\mathrm{AD}$ lesions.

The ability of Lactobacillus species to inhibit certain pathogens has been demonstrated previously. The topical administration of Lactobacillus plantarum to burns in mice has been shown to inhibit Pseudomonas aeruginosa colonization of burns, promote healing, and modify the inflammatory response [21]. A $5 \%$ L. plantarum extract was also effective in reducing skin erythema, repairing skin barrier, and reducing the skin microflora, thereby exhibiting an effective reduction in acne lesion size and erythema [13]. Lactobacillus reuteri protected epidermal keratinocytes from $S$. aureus-induced cell death by competitive exclusion of $S$. aureus from keratinocyte binding sites [22].

Lactobacillus rhamnosus GG, in particular, proved to be extremely efficacious either live or as a lysate in inhibiting the toxic effects of $S$. aureus on epidermal keratinocytes. The authors attributed the beneficial effects to growth inhibition and reduction of bacterial adhesion [23]. Supplementation with Lactobacillus rhamnosus SP1 was also shown to improve adult acne [24].

The present skin care product was also composed of berry seed oils, namely sea buckthorn and blackcurrant oils. The bioprotective, antimicrobial and prebiotic properties of berries have been largely documented [25]. Recent studies have demonstrated that the antimicrobial effects of berries did not affect probi- 
otic species-in particular Lactobacillus strains, exerting their effect only on pathogenic bacteria [26] [27]. The inhibitory and protective effects of berry oils have been attributed to phenolic compounds [25]. The choice of berry seed oils was not only motivated by their antimicrobial and anti-inflammatory properties, but also to serve as a delivery vehicle that keeps the probiotics alive in the skin.

The present study was conducted on a limited number of subjects; a much larger sample size was not planned, but considering the preliminary promising results of our study, larger clinical trials could be planned in a controlled and randomized fashion. As this skin care targets $S$. aureus and aims to restore a balanced microflora on the skin, it would be of high interest to evaluate the effect of the product on the nature and number of bacteria on the affected skin areas. Despite these limitations, we believe that the obtained data provide strong evidence to support the use of this new product in skin care routine in people with atopic skin.

\section{Acknowledgements}

The authors acknowledge the editorial assistance of Emna El Hammi, PhD on behalf of Into-Evidence (Tunisia) and Anne Sirvent, $\mathrm{PhD}$ (Dermscan Group, France) for helpful discussions.

\section{Conflicts of Interest}

The authors declare no conflicts of interest regarding the publication of this paper.

\section{References}

[1] Nutten, S. (2015) Atopic Dermatitis: Global Epidemiology and Risk Factors. Annals of Nutrition \& Metabolism, 66, 8-16. https://doi.org/10.1159/000370220

[2] Eichenfield, L.F., Tom, W.L., Chamlin, S.L., Feldman, S.R., Hanifin, J.M., Simpson, E.L., et al. (2014) Guidelines of Care for the Management of Atopic Dermatitis: Section 1. Diagnosis and Assessment of Atopic Dermatitis. Journal of the American Academy of Dermatology, 70, 338-351. https://doi.org/10.1016/j.jaad.2013.10.010

[3] Avena-Woods, C. (2017) Overview of Atopic Dermatitis. The American Journal of Managed Care, 23, S115-S123.

[4] Malajian, D. and Guttman-Yassky, E. (2015) New Pathogenic and Therapeutic Paradigms in Atopic Dermatitis. Cytokine, 73, 311-318.

https://doi.org/10.1016/j.cyto.2014.11.023

[5] Marks, J.G. and Miller, J.J. (2019) 8-Eczematous Rashes. In: Eds., Lookingbill and Marks' Principles of Dermatology, 6th Edition, Elsevier, London, 95-112.

[6] Drucker, A.M. (2017) Atopic Dermatitis: Burden of Illness, Quality of Life, and Associated Complications. Allergy and Asthma Proceedings, 38, 3-8. https://doi.org/10.2500/aap.2017.38.4005

[7] Lin, Y.T., Wang, C.T. and Chiang, B.L. (2007) Role of Bacterial Pathogens in Atopic Dermatitis. Clinical Reviews in Allergy \& Immunology, 33, 167-177. https://doi.org/10.1007/s12016-007-0044-5

[8] Geoghegan, J.A., Irvine, A.D. and Foster, T.J. (2018) Staphylococcus aureus and 
Atopic Dermatitis: A Complex and Evolving Relationship. Trends in Microbiology, 26, 484-497. https://doi.org/10.1016/j.tim.2017.11.008

[9] Kobayashi, T., Glatz, M., Horiuchi, K., Kawasaki, H., Akiyama, H., Kaplan, D.H., et al. (2015) Dysbiosis and Staphylococcus aureus Colonization Drives Inflammation in Atopic Dermatitis. Immunity, 42, 756-766. https://doi.org/10.1016/j.immuni.2015.03.014

[10] Weidinger, S. and Novak, N. (2016) Atopic Dermatitis. The Lancet (London, England), 387, 1109-1122. https://doi.org/10.1016/S0140-6736(15)00149-X

[11] Eichenfield, L.F., Tom, W.L., Berger, T.G., Krol, A., Paller, A.S., Schwarzenberger, K., et al. (2014) Guidelines of Care for the Management of Atopic Dermatitis: Section 2. Management and Treatment of Atopic Dermatitis with Topical Therapies. Journal of the American Academy of Dermatology, 71, 116-132.

https://doi.org/10.1016/j.jaad.2014.03.023

[12] Roudsari, M.R., Karimi, R., Sohrabvandi, S. and Mortazavian, A.M. (2015) Health Effects of Probiotics on the Skin. Critical Reviews in Food Science and Nutrition, 55, 1219-1240. https://doi.org/10.1080/10408398.2012.680078

[13] Muizzuddin, N., Maher, W., Sullivan, M., Schnittger, S., Mammone, T. (2012) Physiological effect of a probiotic on skin. Journal of cosmetic science, 63, 385-395.

[14] Mottin, V.H.M. and Suyenaga, E.S. (2018) An Approach on the Potential Use of Probiotics in the Treatment of Skin Conditions: Acne and Atopic Dermatitis. International Journal of Dermatology, 57, 1425-1432.

https://doi.org/10.1111/ijd.13972

[15] Rather, I.A., Bajpai, V.K., Kumar, S., Lim, J., Paek, W.K. and Park, Y.H. (2016) Probiotics and Atopic Dermatitis: An Overview. Frontiers in Microbiology, 7, 507. https://doi.org/10.3389/fmicb.2016.00507

[16] Al-Ghazzewi, F.H. and Tester, R.F. (2014) Impact of Prebiotics and Probiotics on Skin Health. Beneficial Microbes, 5, 99-107. https://doi.org/10.3920/BM2013.0040

[17] Notay, M., Foolad, N. and Vaughn, A.R. (2017) Probiotics, Prebiotics, and Synbiotics for the Treatment and Prevention of Adult Dermatological Diseases. American Journal of Clinical Dermatology, 18, 721-732. https://doi.org/10.1007/s40257-017-0300-2

[18] Gueniche, A., Hennino, A., Goujon, C., Dahel, K., Bastien, P., Martin, R., et al. (2006) Improvement of Atopic Dermatitis Skin Symptoms by Vitreoscilla Filiformis Bacterial Extract. European Journal of Dermatology: EJD, 16, 380-384.

[19] Gueniche, A., Bastien, P., Ovigne, J.M., Kermici, M., Courchay, G., Chevalier, V., et al. (2010) Bifidobacterium Longum Lysate, a New Ingredient for Reactive Skin. $E X^{-}$ perimental Dermatology, 19, e1-e8. https://doi.org/10.1111/j.1600-0625.2009.00932.x

[20] Blanchet-Rethore, S., Bourdes, V., Mercenier, A., Haddar, C.H., Verhoeven, P.O. and Andres, P. (2017) Effect of a Lotion Containing the Heat-Treated Probiotic Strain Lactobacillus Johnsonii Ncc 533 on Staphylococcus aureus Colonization in Atopic Dermatitis. Clinical, Cosmetic and Investigational Dermatology, 10, 249-257. https://doi.org/10.2147/CCID.S135529

[21] Valdez, J.C., Peral, M.C., Rachid, M., Santana, M. and Perdigon, G. (2005) Interference of Lactobacillus Plantarum with Pseudomonas Aeruginosa in Vitro and in Infected Burns: The Potential Use of Probiotics in Wound Treatment. Clinical Microbiology and Infection: The Official Publication of the European Society of Clinical Microbiology and Infectious Diseases, 11, 472-479.

https://doi.org/10.1111/j.1469-0691.2005.01142.x 
[22] Prince, T., McBain, A.J. and O’Neill, C.A. (2012) Lactobacillus reuteri Protects Epidermal Keratinocytes from Staphylococcus aureus-Induced Cell Death by Competitive Exclusion. Applied and Environmental Microbiology, 78, 5119-5126. https://doi.org/10.1128/AEM.00595-12

[23] Mohammedsaeed, W., McBain, A.J., Cruickshank, S.M. and O’Neill, C.A. (2014) Lactobacillus rhamnosus gg Inhibits the Toxic Effects of Staphylococcus aureus on Epidermal Keratinocytes. Applied and Environmental Microbiology, 80, 5773-5781. https://doi.org/10.1128/AEM.00861-14

[24] Fabbrocini, G., Bertona, M., Picazo, O., Pareja-Galeano, H., Monfrecola, G. and Emanuele, E. (2016) Supplementation with Lactobacillus rhamnosus sp1 Normalises Skin Expression of Genes Implicated in Insulin Signalling and Improves Adult Acne. Beneficial Microbes, 7, 625-630. https://doi.org/10.3920/BM2016.0089

[25] Lacombe, A. and Wu, V.C.H. (2017) The Potential of Berries to Serve as Selective Inhibitors of Pathogens and Promoters of Beneficial Microorganisms. Food Quality and Safety, 1, 3-12. https://doi.org/10.1093/fqsafe/fyx001

[26] Puupponen-Pimia, R., Nohynek, L., Alakomi, H.L. and Oksman-Caldentey, K.M. (2005) Bioactive Berry Compounds-Novel Tools against Human Pathogens. Ap plied Microbiology and Biotechnology, 67, 8-18. https://doi.org/10.1007/s00253-004-1817-x

[27] Lacombe, A., Wu, V.C., White, J., Tadepalli, S. and Andre, E.E. (2012) The Antimicrobial Properties of the Lowbush Blueberry (Vaccinium Angustifolium) Fractional Components against Foodborne Pathogens and the Conservation of Probiotic Lactobacillus rhamnosus. Food Microbiology, 30, 124-131.

https://doi.org/10.1016/j.fm.2011.10.006 